\title{
Soft Nanopatterning on Light-Emitting Inorganic-Organic Composites**
}

\author{
By Luana Persano, Sandra Molle, Salvatore Girardo, Antonio A. R. Neves, Andrea Camposeo, Ripalta Stabile, \\ Roberto Cingolani, and Dario Pisignano*
}

In this work we demonstrate the nanopatterning of nanocomposites made by luminescent zinc oxide nanoparticles and lightemitting conjugated polymers by means of soft molding lithography. Vertical nanofluidics is exploited to overcome the polymer transport difficulties intrinsic in materials incorporating nanocrystals, and the rheology, fluorescence, absolute quantum yield, and emission directionality of the nanostructured composites are investigated. We study the effect of patterned gratings on the directionality of light emitted from the nanocomposites, finding evidence of the enhancement of forward emitted light, due to the printed wavelength-scale periodicity. These results open new possibilities for the realization of nanopatterned devices based on hybrid organic-inorganic systems.

\section{Introduction}

Nanocomposite systems ${ }^{[1]}$ consisting of a polymer matrix filled by inorganic nanoparticles exhibit tailored or improved mechanical, ${ }^{[2]}$ thermal, ${ }^{[3]}$ catalytic $^{[4]}$ or optical ${ }^{[5]}$ properties. In particular, the incorporation of micro- or nanocrystals in conjugated polymers ${ }^{[5 a, c, e, g, i]}$ allows one to exploit the wide tunability, ease of processing and structural flexibility of organics, together with the optical and thermal stability of inorganic light-emitting fillers for the specific design of broadly tunable optically active materials. In our previous work, ${ }^{[5 i]}$ we studied how the organic-inorganic interaction can be often described through a dipole-surface scheme, determining the characteristic activation energies of the exciton recombination routes in the nanocomposite. However, a crucial issue for the realization of nanocomposite-based optoelectronic devices still is the development of suitable lithographic technologies for wavelength-scale (sub-micrometer) patterning. To date, patterning of hybrid nanocomposites embedding luminescent nanocrystals has been accomplished by electronbeam, ${ }^{[6]}$ optical ${ }^{[7]}$ and imprint lithographies, ${ }^{[8]}$ by exploiting optically inert matrices such as methacrylate polymers and copolymers, ${ }^{[6,7 b, 8 b]}$ epoxy-based photoresists ${ }^{[7 a]}$ and titania

[*] Dr. D. Pisignano, ${ }^{+}$Dr. L. Persano, S. Molle, S. Girardo, Dr. A. A. R. Neves, Dr. A. Camposeo, R. Stabile, Prof. R. Cingolani

NNL, National Nanotechnology Laboratory of Instituto Nazionale di Fisica della Materia-Consiglio

Nazionale delle Ricerche, c/o Distretto Tecnologico ISUFI via Arnesano, I-73100 Lecce (Italy)

E-mail: luana.persano@unile.it

[+] Present address: Istituto Superiore di Formazione Interdisciplinare (ISUFI), Università del Salento, Lecce (Italy)

[**] We gratefully acknowledge the financial support from the Regional Strategic Project 'Ponamat', from the Italian Institute of Technology, and from the Italian Ministry of University and Research through the FIRB project RBIP06SH3W. pre-polymers. ${ }^{[8 \mathrm{a}]}$ Instead, notwithstanding the great importance of these materials for both fundamental studies and applications, ${ }^{[5 \mathrm{a}, \mathrm{c}, \mathrm{e}, \mathrm{g}, \mathrm{i}]}$ nanopatterning of composites with nanocrystals incorporated in light-emitting organics is basically unexplored, likely due to the more difficult processability induced by the limited solubility and relatively poor thermoplastic behaviour of many conjugated polymers. Here we demonstrate the nanopatterning of such an important class of composites by soft moulding (SM) lithography, achieving resolutions up to a few hundreds of nanometres on several light-emitting polymers embedding zinc oxide particles [molecular structures (1-4) and crystalline structure of $\mathrm{ZnO}$ displayed in Fig. 1].

\section{Results and Discussion}

The SM, or capillary force lithography, ${ }^{[9]}$ combines procedures and advantages of soft ${ }^{[10]}$ and nanoimprint ${ }^{[11]}$ methods. A scheme of our process is shown in Figure 2a-d. Once an elastomeric mould is put in contact with the surface of the nanocomposite film (Fig. 2a), the overall system is driven above the softening temperature of the polymer matrix (Fig. 2b). Widely employed in soft lithographies, ${ }^{[10]}$ low Young modulus elastomeric stamps are particularly advisable for texturing nanocomposites, whose surface roughness can be substantially increased by the incorporated nanocrystals and their possible aggregates, hence requiring remarkable shortscale $(<10 \mu \mathrm{m})^{[12]}$ conformability of the moulds to obtain effective pattern transfer. The composite starts to wet the lateral walls of the recessed features in the mould up a filled height depending on the heating time (Fig. 2b, c). Afterwards, the system is driven below the matrix glass transition temperature $\left(T_{\mathrm{g}}\right)$, and the mould is detached from the surface (Fig. 2d), which turns out to be textured with the same pattern 
(1)<smiles>CCCC1(CCC)c2ccccc2-c2ccccc21</smiles><smiles>CCCCCCCCn1c2ccccc2c2ccccc21</smiles>

(3)

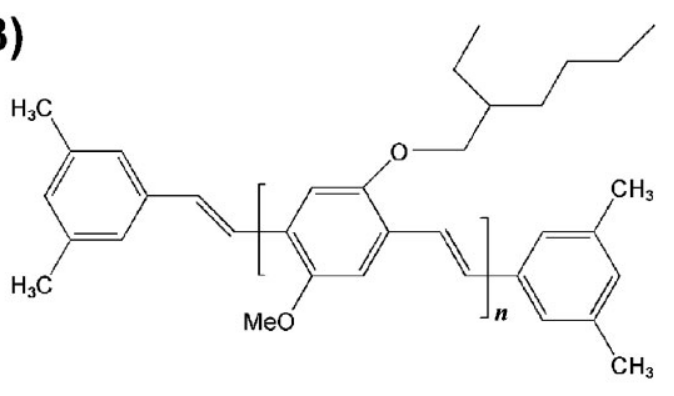

(2)<smiles>Cc1ccc(-c2ccc3c(c2)C(C)(C)c2cc(C(C)C)ccc2-3)c2nsnc12</smiles>

(4)

(5)

Figure 1. Molecular structures of conjugated polymers employed in light-emitting nanocomposites: (1) poly[(9,9-dioctylfl uorenyl-2,7-diyl)-alt-co-(9-hexyl3,6-carbazole)], (2) poly[(9,9-dioctylfluorenyl-2,7-diyl)-co-(1,4-benzo-\{2,1',3\}-thiadiazole)], (3) MEH-PPV and (4) poly \{[2-methoxy-5-(2-ethylhexyloxy)-1,4-(1cyanovinylenephenylene)]-co-[2,5-bis ( $N, N^{\prime}$-diphenylamino)-1,4-phenylene $\left.]\right\}$ and $\mathrm{ZnO}$ crystal structure $(5$, smaller dots $=\mathrm{Zn}$ atoms, larger dots $=\mathrm{O}$ atoms).

(a)
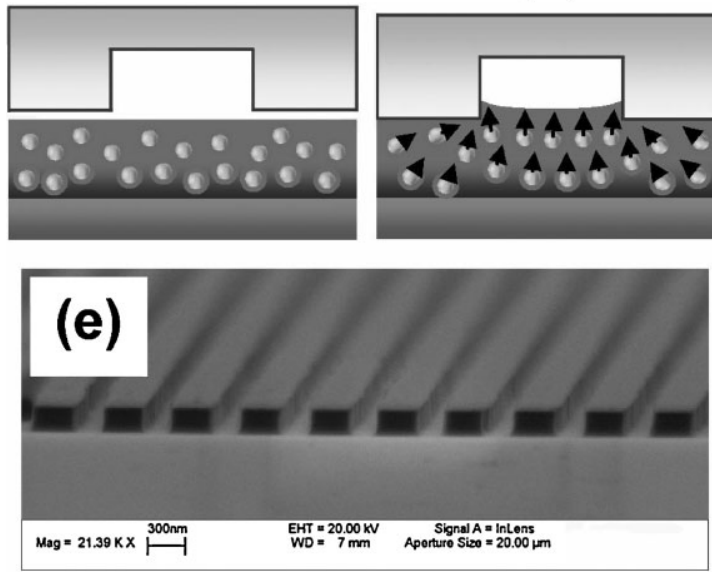

(b)
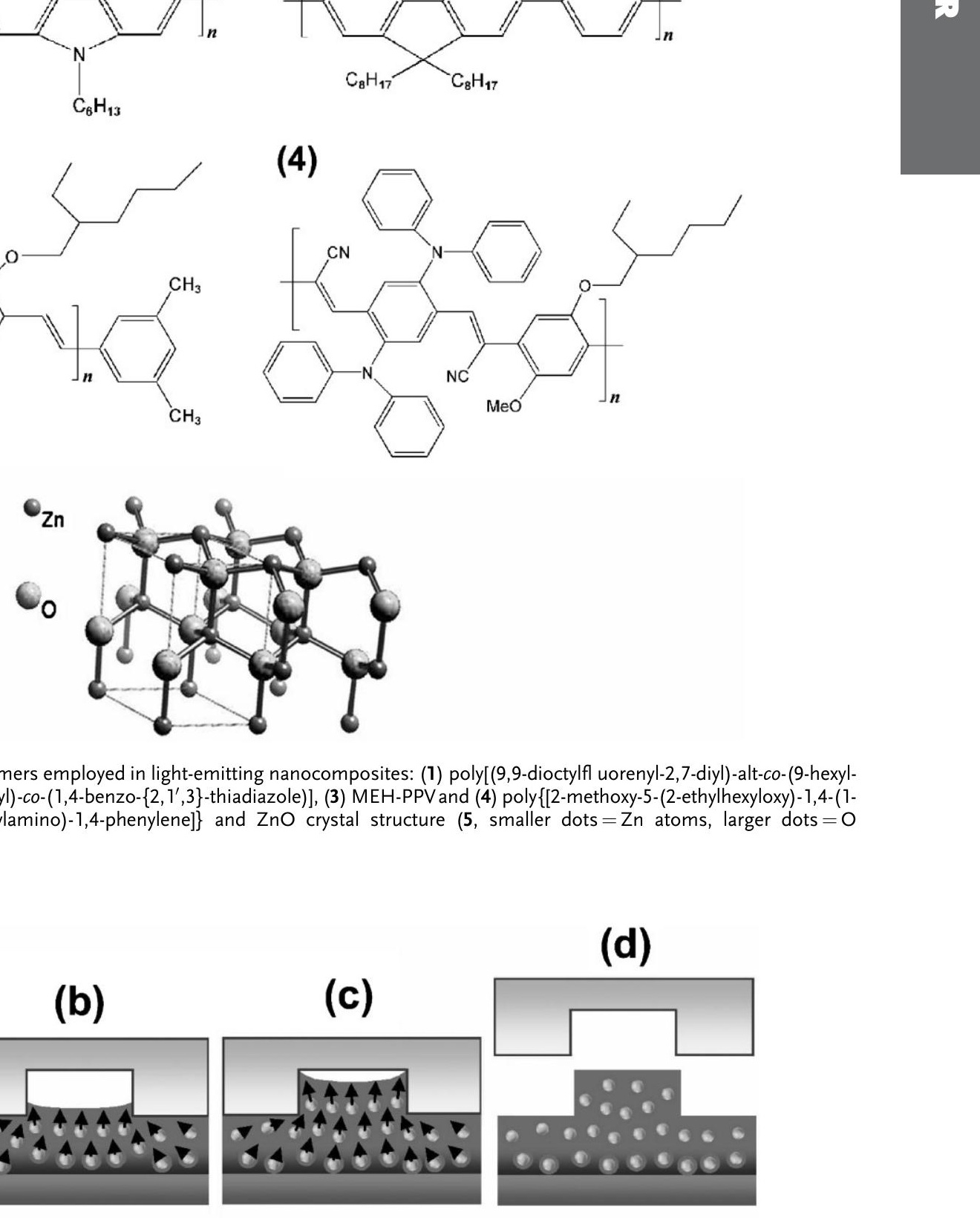

(d)

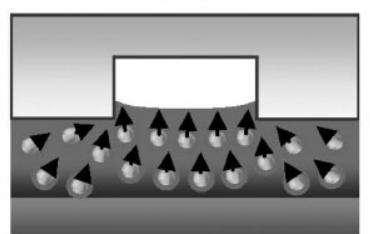
.

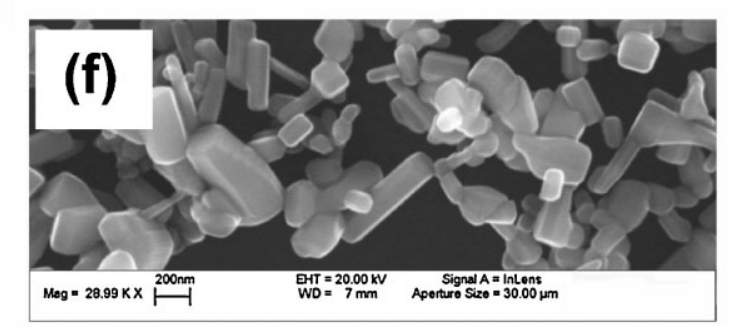

Figure 2. a)-d) Schematic diagram of SM process on nanocomposites (features not in scale). a) Mould-composite contact, b) heating above softening temperature, c) capillarity driven nanofluidic filling, d) cooling down to room temperature and mould separation. e) Cross-sectional view of a 560 nmperiod master template. f) Employed ZnO nanoparticles imaged by SEM. 
as the starting master template [scanning electron microscopy (SEM) picture displayed in Fig. 2e]. Our transferred patterns include gratings with period $(\Lambda)$ between $500 \mathrm{~nm}$ and a few micrometres, on nanocomposites embedding $\mathrm{ZnO}$ particles of size in the range of 100-600 nm (Fig. 2f).

In SM, the vertical transport of the composite relies on capillarity effects undergone by the polymer component, the main driving force being the Laplace pressure, $P_{\mathrm{L}}=2 \gamma \cos \Theta / R$, where $\gamma, \Theta$ and $R$ indicate the fluid-airsurface tension, the contact angle between the liquid and the surface of the micro- or nanochannels given by the recessed mould features, and the hydraulic radius of the capillary, respectively. In the simplified case of viscous forces predominant over inertial forces and of laminar flow for Newtonian melts, ${ }^{[13]}$ the time required to fill the voids of mould up to the height, $z$, is given by the following equation: ${ }^{[9]}$

$t=\frac{2 \eta z^{2}}{\gamma R \cos \theta}$

where $\eta$ indicates the viscosity of the rising composite. The Poiseuille flow is therefore strongly affected by $\eta$. As in many organic materials the viscosity above $T_{\mathrm{g}}$ exhibits a dramatic decrease because of the increased molecular mobility, ${ }^{[14]}$ the increase in temperature is effectively exploited in SM to allow the filling of the pattern in a few minutes. However, since the presence of incorporated particles can significantly disfavour the micro- or nanofluidic molecular flow, longer heating intervals (up to two to three times) and higher temperatures (by about $50^{\circ} \mathrm{C}$ ) are required for SM on composites with respect to the corresponding purely polymeric materials, as we find for all our investigated systems. The choice of the processing temperature is particularly critical in the case of light-emitting nanocomposites, since, while low temperatures can cause incomplete or too slow capillary penetration, excess heating may lead to (i) very low viscosity of the organic component, thus not being able to vertically carry inorganic nanoparticles and (ii) full, irreversible degradation of the luminescence properties mainly induced by oxygen incorporation and substitution ${ }^{[15]}$ during high-temperature soft lithography. For the optimal choice of the process parameters, an in-depth assessment of the rheological behaviour of the nanocomposite films is therefore particularly important.

For this reason, we analyse the thermo-mechanical response of the prototypical light-emitting blend, poly[2-methoxy-5-(2ethylhexyloxy)-1,4-phenylene-vinylene] (MEH-PPV)/poly (methylmethacrylate) (PMMA) and of its composite with $\mathrm{ZnO}$ particles. Figure 3 displays the dynamic storage $\left(G^{\prime}\right)$ and loss $\left(G^{\prime \prime}\right)$ moduli (a) of PMMA, (b) of the polymeric blend and (c) of the nanocomposite, respectively, measured at $170{ }^{\circ} \mathrm{C}$ for frequencies $(\omega)$ consistent with the values of shear-rates of viscous systems at the entrance of micro- or nanochannels. ${ }^{[16]}$ A transition from solid-like $\left(G^{\prime}>G^{\prime \prime}\right)$ to liquid-like $\left(G^{\prime}<G^{\prime \prime}\right)$ behaviour is clearly appreciable from the intersection of the

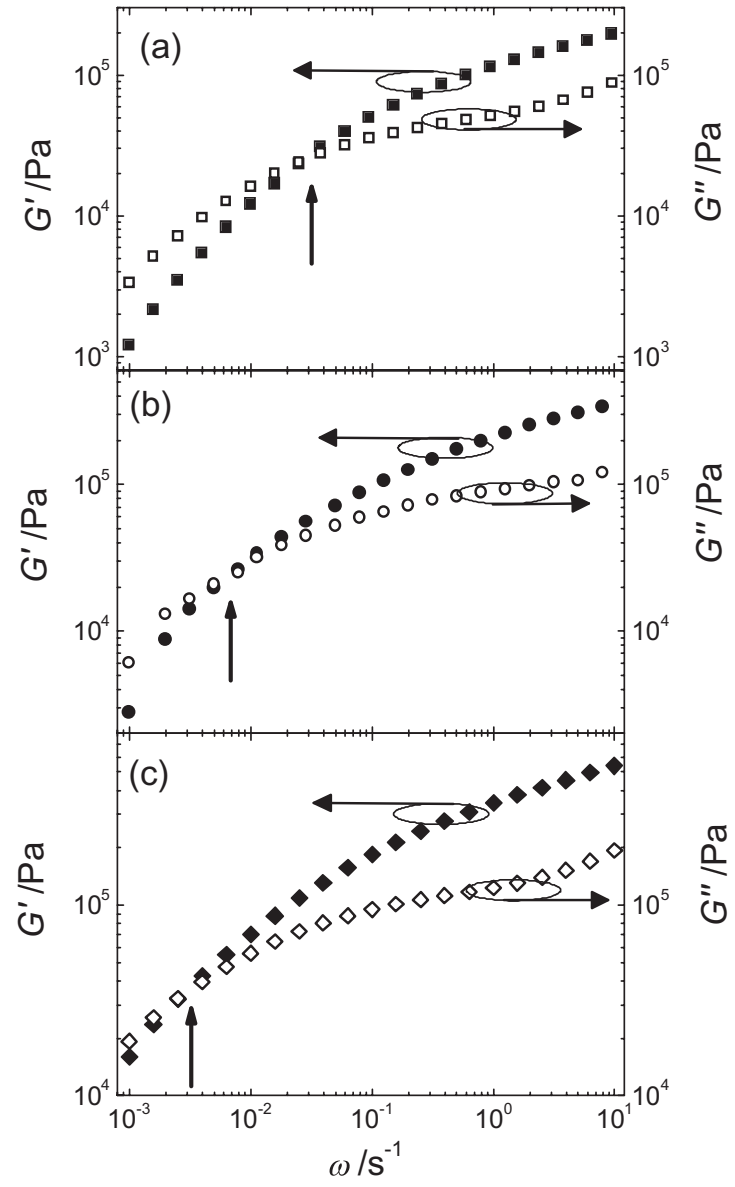

Figure 3. Storage modulus, $G^{\prime}$ (full symbols, left vertical scale), and loss modulus, $C^{\prime \prime}$ (empty symbols, right scale) versus frequency, $\omega$, for PMMA a), MEH-PPV/PMMA b), and $\mathrm{ZnO}$ nanocomposite c).

linear viscoelastic moduli upon decreasing $\omega$, with crossover frequency, $\omega_{\mathrm{c}}$ (for which $G^{\prime}$ equals $G^{\prime \prime}$ ) of $3 \times 10^{-2}, 6 \times 10^{-3}$ and $3 \times 10^{-3} \mathrm{~s}^{-1}$ for the PMMA, the polymeric blend and the nanocomposite, respectively. Therefore, the inclusion of inorganic nanoparticles in the polymer matrix doubles the longest relaxation time $\left(\tau=\omega_{\mathrm{c}}^{-1}\right)^{[17 \mathrm{a}]}$ in the system, moreover increasing the absolute values of the elastic modulus, and decreases the slope of the $G^{\prime}$ and $G^{\prime \prime}$ curves (Fig. $3 \mathrm{~b}$ and c). A similar behaviour is observed in a number of composite systems such as fluoroelastomers ${ }^{[17 a]}$ and thermoplastics ${ }^{[17 b-f]}$ incorporating organoclays, and concentrated suspensions of hard spheres, ${ }^{[17 \mathrm{~g}]}$ and attributed to the reduction of local mobility of polymeric chains as consequence of strong fillerpolymer interactions resulting in physically networked structures, or in shear-induced supramolecular orientation. The system viscosity is enhanced upon addition of the nanocrystals as well (Fig. 4). With respect to PMMA, $\eta$ is more than four times larger in the nanocomposite including $\mathrm{ZnO}$ particles, which provided resistance to melt flow and deformations. Furthermore, the ratio of the lost to stored energy, $\tan \delta=G^{\prime \prime}$ | $G^{\prime}$ is decreased by the inclusion of inorganic fillers in the polymeric blends (inset of Fig. 4), which is related to an 


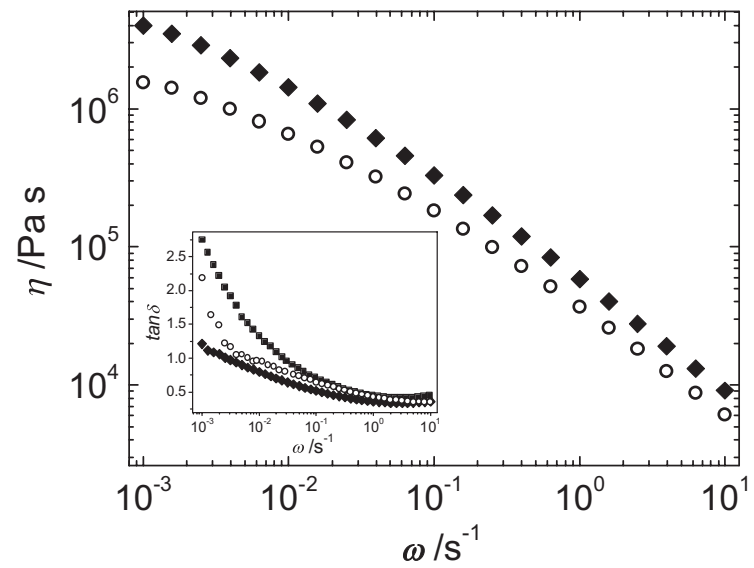

Figure 4. Frequency dependence of viscosity, $\eta$, of PMMA (full squares), MEH-PPV/PMMA (empty circles) and $\mathrm{ZnO}$ nanocomposite (full diamonds). Inset: frequency dependence of the dissipation coefficient, $\tan \delta$, for the three materials (same used symbols).

enhancement of the elastic, solid-like character of the material. However, a shear-thinning behaviour is appreciable in all the investigated range of $\omega$ (Fig. 4), and particularly for the composite system, from which high-temperature patterning of the nanocomposites can benefit. ${ }^{[17 \mathrm{c}]}$ In particular, since SM heating must be longer than the characteristic time-scale of structural rearrangement of the melt, the composite material has to be kept at high temperature for longer times ( 15 instead of $5 \mathrm{~min}$ ) to allow its terminal flow to determine the irreversible deformation and hence the pattern transfer from the mould.

This is shown in Figure 5, displaying the micrographs of gratings with $\Lambda=4 \mu \mathrm{m}$ on the mould template (bright field image in Fig. 5a) and on printed nanocomposites of $\mathrm{ZnO}$ particles embedded in the four light-emitting polymers, 1-4 (fluorescence images in Fig. 5b-e, respectively). The good fidelity of the mould and the large patterned area are appreciable from the comparison of Figure 5a and b-e. In particular, the obtained periodicity faithfully reproduces that of the mould, and the two dimensional view and the crosssection of the final nanocomposite gratings, imaged by atomic force microscopy (AFM) in Figure 5f and $g$, show a welldefined pattern, clearly indicating that the achievable resolution can be further reduced, depending on the starting master. A direct comparison between a master with $\Lambda=560 \mathrm{~nm}$, fabricated by electron-beam lithography (EBL), and the resulting nanocomposite pattern allows one to appreciate that the final periodicity and width of the features replicate those of the template with good fidelity even in the sub- $\mu \mathrm{m}$ scale (Fig. 6). An aspect-ratio loss of the order of $20 \%$ with respect to the master is observable in the printed sample because of an incomplete filling of the recessed features of the mould, which can be reduced by increasing the heating interval during SM or by slightly decreasing the nanoparticles amount in the composite film. The minor topographic inhomogeneities observable in the AFM images of the nanocomposites correspond to the regions of luminescence contrast due to
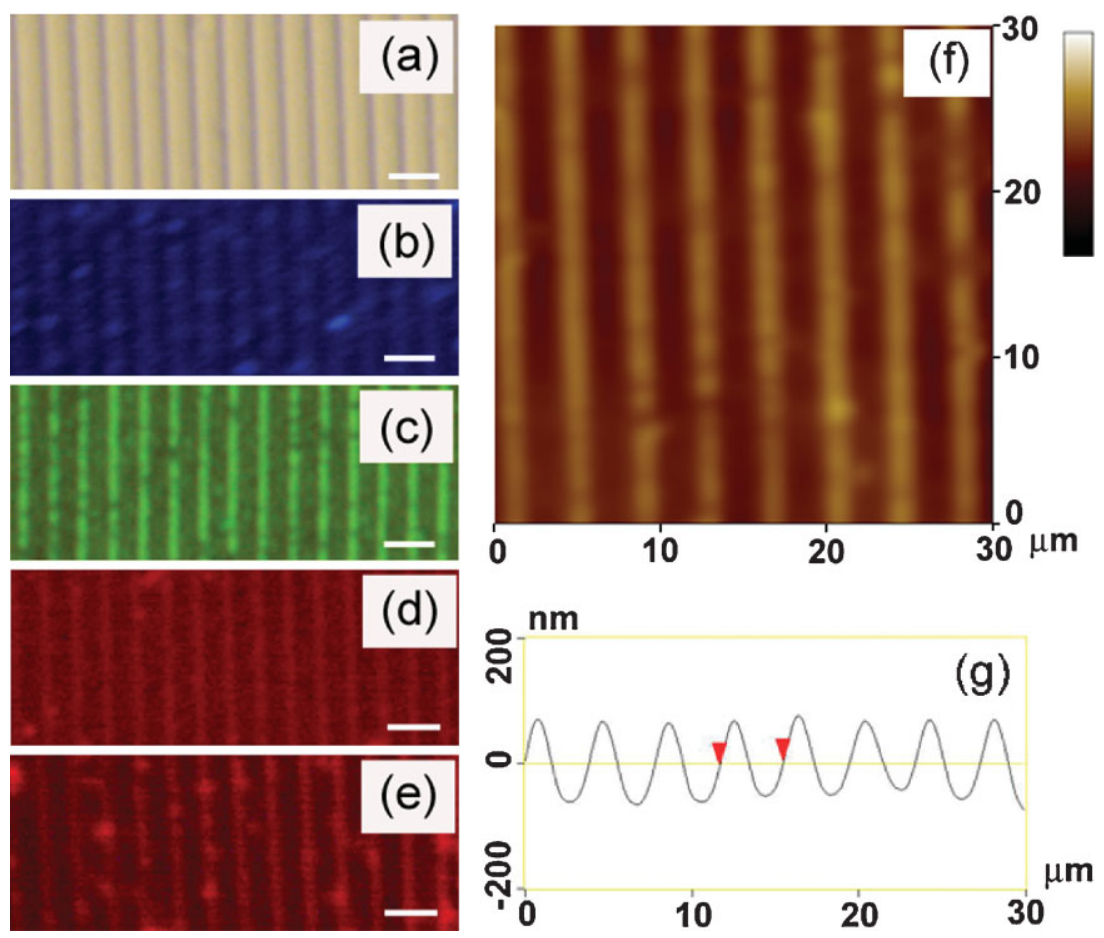

Figure 5. Bright field optical micrograph of $4 \mu \mathrm{m}$-period mould a), and corresponding SM pattern transfer to nanocomposites of $\mathrm{ZnO}$ and conjugated polymers (1-4), imaged by fluorescence microscopy (b-e, respectively). Marker $=5 \mu \mathrm{m}$. e) AFM two-dimensional view of the nanocomposite grating. f) Corresponding cross-section. Vertical scale from zero to $500 \mathrm{~nm}$. 

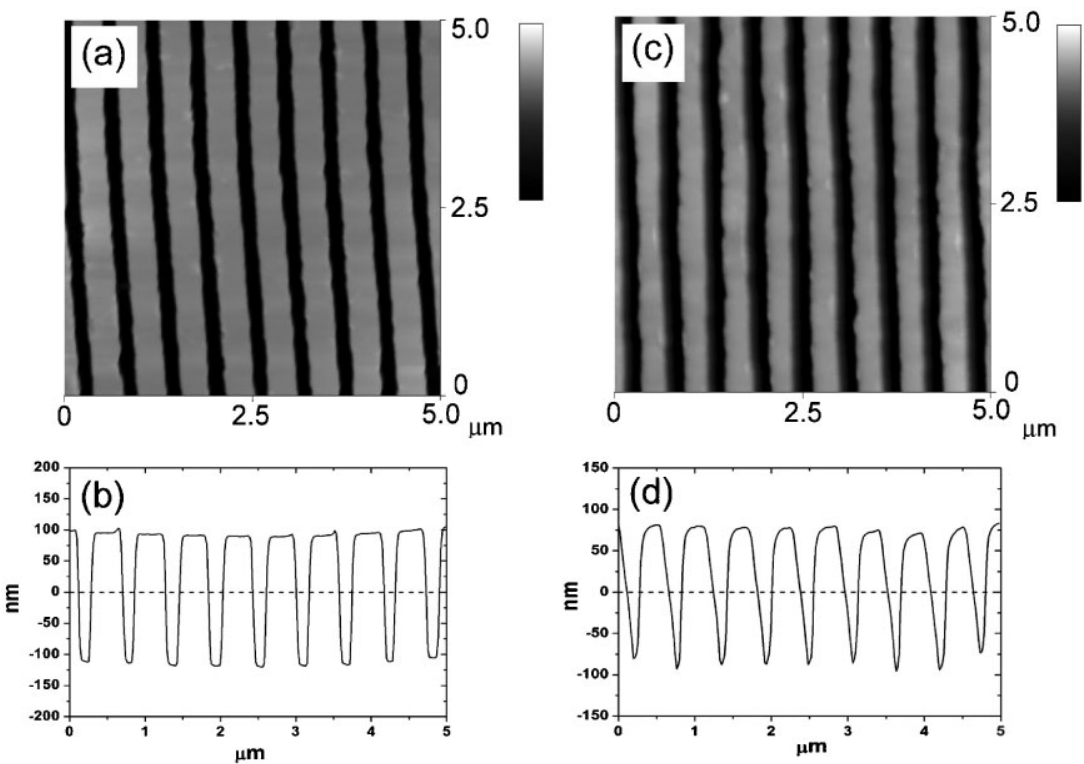

Figure 6. AFM view of a $560 \mathrm{~nm}$-period master a), vertical scale from zero to $300 \mathrm{~nm}$, and master cross-sectional profile b), together with the corresponding patterned nanocomposite with MEH-PPV c) vertical scale from zero to $200 \mathrm{~nm}$ and resulting cross-section d). Typical patterned features in experiments with this master exhibit height from 15 to 20 to more than $160 \mathrm{~nm}$, and full width at half maximum of about $400 \mathrm{~nm}$, faithfully replicating the width of the master features.

local microscale aggregations of particles, analogously with experiments on patterning of nanocrystals films. ${ }^{[5 \mathrm{f}]}$

In order to assess the retained luminescence properties of patterned nanocomposites, we collected the photoluminescence (PL) spectra before and after the lithography process (Fig. 7), and investigated for the first time the PL efficiency, $\Phi$, achievable with nanocrystals-conjugated polymer donoracceptor systems. For all the studied composites, the integrated PL spectra show slight variation in the emission peak wavelength, $\lambda(\leq 5 \%)$, and a change of the full width at half maximum, $\Delta \lambda$, up to $40 \%$ (Table 1 ). In some light-emitting polymers, excited by the embedded inorganic particles via non-radiative energy transfer, the spectra exhibit an enhance-

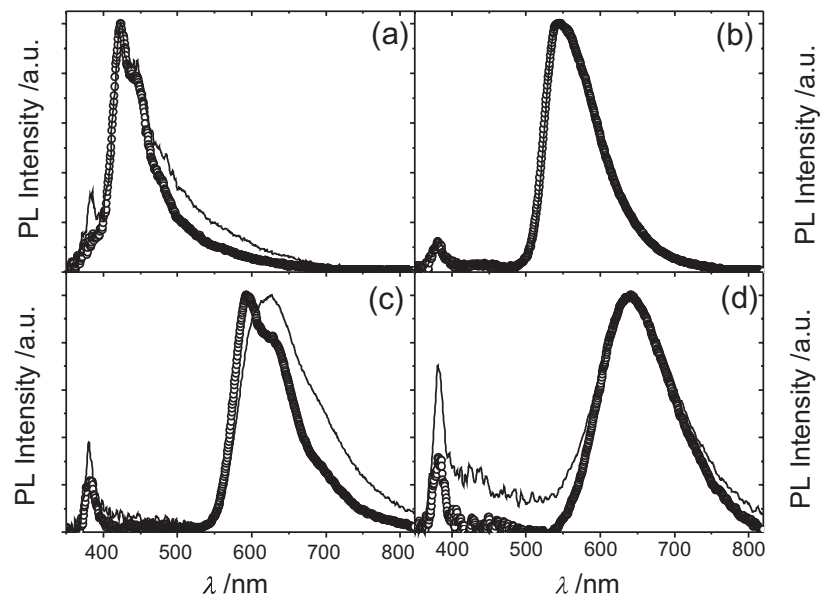

Figure 7. Integrated PL spectra before (empty circles) and after (superimposed lines) SM nanopatterning. From a) to d), spectra correspond to nanocomposites with polymers from 1 to 4 , respectively. ment of the low-energy vibronic replicas or tails, suggesting oxidative reduction of the conjugation length or excitonic migration and relaxation to excimer species. ${ }^{[18]}$ Nonetheless, the absolute quantum yield after SM is still generally larger than $10-15 \%$ for all the composites, whose figure of merit is fully valid for the eventual realization of nanopatterned lightemitting devices (Table 1). ${ }^{[19]}$

To this aim, we study the effect of patterned gratings on the directionality of light emitted from the nanocomposites. The angle-resolved PL spectra of a film with $\mathrm{ZnO}$ particles and MEH-PPV structured with a $560 \mathrm{~nm}$ period grating are shown in Figure 8. The presence of the periodic corrugation in the nanocomposite enhances the light emitted towards certain forward angles $(\Psi)$, due to the printed, one-dimensional wavelength-scale periodicity. In such linear distributed feedback microcavities, the angle-dependent emission wavelength, $\lambda_{\Psi}$, relates to the effective refractive index of the waveguide mode, $n_{\text {eff }}$, and to the diffraction order inside the luminescent slab according to the conservation of the in-plane component of the wave vector of the emitted light. ${ }^{[20]}$ By this relation, we

Table 1. Emission spectral properties, peak wavelength $(\lambda)$ and full width at half maximum $(\Delta \lambda)$ and PL quantum yields $(\Phi)$ of the nanocomposites, before and after SM.

\begin{tabular}{lccccccc}
\hline Composite & \multicolumn{3}{c}{ Before SM } & & \multicolumn{3}{c}{ After SM } \\
\cline { 2 - 3 } \cline { 7 - 8 } & $\lambda[\mathrm{nm}]$ & $\Delta \lambda[\mathrm{nm}]$ & $\boldsymbol{\Phi}$ & & $\lambda[\mathrm{nm}]$ & $\boldsymbol{\Delta} \lambda[\mathrm{nm}]$ & $\boldsymbol{\Phi}$ \\
\hline $\mathbf{1}$ & 423 & 52 & $46 \pm 4$ & & 423 & 63 & $24 \pm 6$ \\
$\mathbf{2}$ & 545 & 82 & $92 \pm 3$ & 543 & 79 & $72 \pm 7$ \\
$\mathbf{3}$ & 593 & 87 & $35 \pm 4$ & 627 & 119 & $17 \pm 4$ \\
$\mathbf{4}$ & 641 & 115 & $29 \pm 1$ & 648 & 129 & $20 \pm 6$ \\
\hline
\end{tabular}




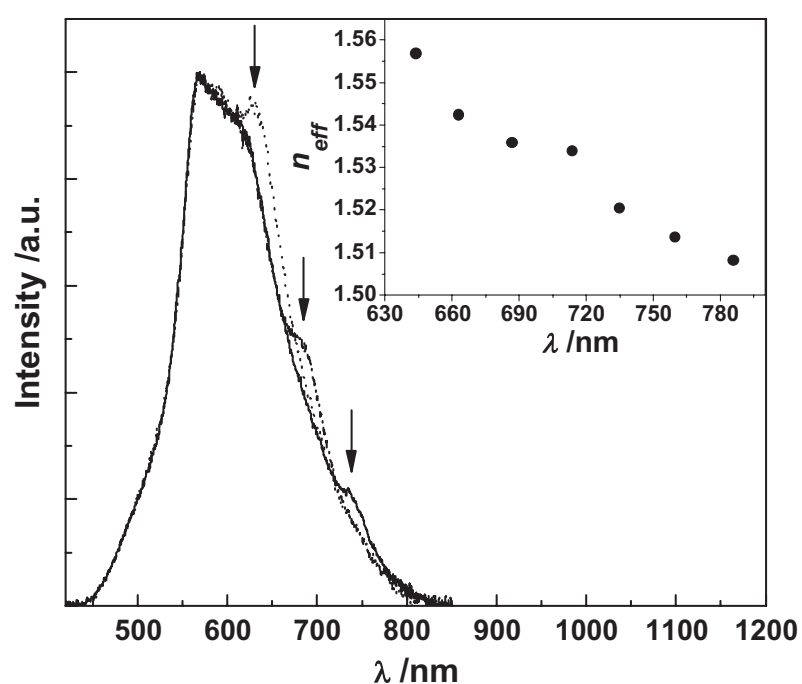

Figure 8. a): $\mathrm{PL}$ spectra from a $560 \mathrm{~nm}$-period patterned nanocomposite (with MEH-PPV), at collection angle: $12^{\circ}$ (solid line), $18^{\circ}$ (dashed line) and $24^{\circ}$ (dotted line). The vertical arrows evidence the angular dependence of the emission peaks induced by the imprinted grating. Inset: wavelength dependence of $n_{\text {eff. }}$

estimate the wavelength dependence of $n_{\text {eff }}$ of the nanostructured light-emitting composite, which decreases from 1.56 to 1.51 upon increasing $\lambda_{\Psi}$ from 644 to $786 \mathrm{~nm}$ (inset of Fig. 8).

The specific advantages of this approach over other technologies for patterning nanocomposites ${ }^{[6-8]}$ are numerous. The SM is not affected by multiple reflections, scattering, and in situ diffraction due to embedded nanoparticles, ${ }^{[7 \mathrm{a}]}$ which, besides well-know mask diffraction effects, can limit the resolution and the exposure homogeneity achievable with optical methods when applied to nano-scale heterogeneous materials. The method is parallel, and cheap as it does not need any exposure or pressing set-up. In SM, the penetration of the polymer into the recessed features of the mould is driven by capillarity effects, thus being marginally affected by problems of difficult polymer transport, which can be relevant under the pressure applied by hard moulds during nanoimprinting, and further enhanced by the presence of nanoparticles suppressing the molecule flow, ${ }^{[21]}$ as here demonstrated. Concomitantly, SM is immune from pattern shrinkage or distortions, induced by the solvents employed by other soft lithography techniques. We believe that a more controlled atmosphere for film preparation and processing can lead to an even better preservation of the optical properties of light-emitting composites, with respect to the here reported results.

\section{Conclusions}

We demonstrate structuring of light-emitting nanocomposite materials including $\mathrm{ZnO}$ particles and conjugated polymers, with resolution up to wavelength-scale. The SM technology allows one to overcome the flow difficulties induced by embedding nanocrystals in polymer compounds. These results open new possibilities for the realization of nanopatterned devices based on hybrid organic-inorganic systems. Texturing composites with high (sub-500 nm) spatial resolution will enable tailoring the device emission resulting from donor-acceptor energy transfer by lithographic filtering of the nanoparticles able to penetrate in the mould template by vertical nanofluidics.

\section{Experimental}

Materials: For the preparation of the nanocomposites, the following light-emitting conjugated polymers were used, purchased from American Dye Source Inc. (Quebec, Canada): (1) poly[(9,9-dioctylf uorenyl-2,7-diyl)-alt-co-(9-hexyl-3,6-carbazole)], (2) poly[(9,9-dioctylfluorenyl-2,7-diyl)-co-(1,4-benzo-\{2,1',3\}-thiadiazole)], (3) MEH-PPV and (4) poly\{[2-methoxy-5-(2-ethylhexyloxy)-1,4-(1-cyanovinylenephenylene)]-co-[2,5-bis( $N, N^{\prime}$-diphenylamino)-1,4-phenylene]\}. Zinc oxide particles were purchased by Sigma-Aldrich, Inc. PMMA (Sigma-Aldrich) was also employed as blending component in the composites aiming to increase the radiative decay efficiency by reducing the formation of nonradiative recombination centres in the conjugated polymers and to improve the overall photostability ${ }^{[\mathrm{h}]}$. Solutions were prepared by dispersing polymers and $\mathrm{ZnO}$ particles in toluene (J.T. Baker, Phillipsburg, NJ), with relative concentrations ( $\mathrm{ZnO} /$ conjugated polymer) between $3: 2$ and $1: 4$ in weight. PDMS (Sylgard 184, Dow Corning, Midland, MI, USA), trimethylsiloxy-terminated vinylmethylsiloxane-dimethylsiloxane (VDT-731, ABCR, Karlsruhe, Germany), platinum divinyltetramethyldisiloxane (ABCR), 2,4,6,8-tetramethyltetravinylcyclotetrasiloxane (SigmaAldrich) and methylhydrosiloxane-dimethylsiloxane (HMS-301, ABCR) were used for the realization of elastomeric moulds.

Lithography: A set of master structures made by parallel stripes with period ranging from $560 \mathrm{~nm}$ to $4 \mu \mathrm{m}$ was fabricated. Submicrometre period gratings were obtained by EBL on silicon wafers, performed with a Raith 150 system at an acceleration energy of $20 \mathrm{KeV}$, lift-off and subsequent reactive ion etching by a $\mathrm{CF}_{4} / \mathrm{Ar}$ mixture. Micrometre-scale masters were realized by optical lithography on $\mathrm{Si} / \mathrm{SiO}_{2}$ substrates, with an EVG 620 mask aligner. After the deposition of standard photoresist, UV-exposure and development, we etched the thermal dioxide by using $\mathrm{NH}_{4} \mathrm{~F} / \mathrm{HF} / \mathrm{H}_{2} \mathrm{O}$ $(6.6 \mathrm{~g}: 1.6 \mathrm{~mL}: 10 \mathrm{~mL})$. The so obtained $\mathrm{SiO}_{2}$ mask was then employed to etch Silicon by $5.9 \mathrm{M}$ solutions of $\mathrm{KOH}$ at $80^{\circ} \mathrm{C}$. PDMS and $h$-PDMS moulds were then obtained from the masters according to standard procedures [10]. Nanocomposite films were spin-cast onto cleaned quartz substrates from the toluene solutions, at $1600 \mathrm{rpm}$ for $60 \mathrm{~s}$, providing a uniform film thickness of about $350 \mathrm{~nm}$. SM was carried out by placing the moulds on the composite films under their own weight, and heating the samples at $170^{\circ} \mathrm{C}$ in glove-box nitrogen atmosphere (oxygen ppm $<10$ ), for about $15 \mathrm{~min}$. Generally, PDMS moulds led to better patterning results due to higher conformability to local surface dishomogeneities in the nanocomposite films.

Rheological Characterization: Rheological measurements were carried out by a rotational rheometer (TA Instruments Inc., New Castle, DE, USA). Samples of PMMA, MEH-PPV/PMMA and composites including $\mathrm{ZnO}$ particles with relative concentration of $60 \%$ with respect to MEH-PPV were prepared as disks of $1 \mathrm{~mm}$ thickness and $25 \mathrm{~mm}$ diameter. They were studied using a parallel plate geometry, at $T=170^{\circ} \mathrm{C}$ under nitrogen atmosphere. The linear viscoelasticity region for the investigated materials was determinated by amplitude sweep experiments.

AFM Characterization: The morphological characterization of the nanopatterned surfaces was carried out by tapping AFM in air, by using a Nanoscope III controller with a Digital Instruments Multimode head, 
integrated with a J-scanner (maximum scan size of $90 \times 90 \mu \mathrm{m}^{2}$ ). Phosphorous-doped Si tips were employed with an 8-10 nm nominal curvature radius and a resonant frequency of 160 and $280 \mathrm{kHz}$.

Optical Measurements: Fluorescence micrographs of the nanocomposite gratings were collected by a Leica MZ16FA stereomicroscope coupled to a $3264 \times 2448$ pixels camera (Leica DFC490), under excitation by a Hg lamp. The PL spectra of the nanocomposite films before and after patterning were measured by a fiber-coupled monochromator (TRIAX 320, Jobin Yvon), equipped with a charge coupled device (Jobin Yvon), and exciting the materials with a $\mathrm{He}-\mathrm{Cd}$ laser $(\lambda=325 \mathrm{~nm})$. Angle-resolved PL measurements were carried out by collecting photons emitted in a small solid angle $\left(\cong 10^{-3} \mathrm{rad}\right)$ around each value of the collection angle $(\Psi)$, measured by moving in the plane perpendicular to the nanopatterned grooves. The luminescence absolute quantum efficiency $(\Phi)$ was measured by determining the number of PL photons emitted per absorbed photon, placing samples in an integrating sphere, and accounting for the excitation light which is not absorbed by the sample at its first incidence, and is absorbed after successive reflections on the reflecting sphere surface [19]:

$\Phi=\frac{P_{1}-(R+T) P_{2}}{(1-R-T) X_{L}}$

In the previous expression, $R$ and $T$ indicate the sample reflectance and transmittance, respectively, $P_{1}$ and $P_{2}$ are the PL signals measured with the laser beam incident on the sample and on the integrating sphere, respectively, and $X_{\mathrm{L}}$ stands for the excitation signal, measured with the laser incident on the sphere and without sample. All the optical measurements were carried out at room temperature.

Received: February 18, 2008 Revised: May 26, 2008

Published online: September 1, 2008

[1] a) C. Sanchez, B. Lebeau, F. Chaput, J. P. Boilot, Adv. Mater. 2003, 15, 1969. b) A. Maity, M. Biswas, J. Ind. Eng. Chem. 2006, 12, 311. c) A. C. Balazs, T. Emrick, T. P. Russell, Science 2006, 314, 1107.

[2] G. M. Russo, G. P. Simon, L. Incarnato, Macromolecules 2006, 39, 3855.

[3] R. Gangopadhyay, A. De, Chem. Mater. 2000, 12, 608.

[4] K. Esumi, H. Houdatsu, T. Yoshimura, Langmuir 2004, 20, 2536.

[5] a) V. L. Colvin, M. C. Schlamp, A. P. Alivisatos, Nature 1994, 370, 354. b) W. Caseri, Macromol. Rapid Commun. 2000, 21, 705. c) N. Tessler, V. Medvedev, M. Kazes, S. Kan, U. Banin, Science 2002, 295, 1506. d) W. H. Huynh, J. J. Dittmer, A. P. Alivisatos, Science 2002, 295, 2425. e) L. Bakueva, S. Musikhin, M. A. Hines, T.-W. F. Chang, M. Tzolov, G. D. Scholes, E. H. Sargent, Appl. Phys. Lett. 2003, 82, 2895. f) Y. Wang, Z. Tang, M. A. Correa-Duarte, L. M. Liz-Marzán, N. A. Kotov, J. Am. Chem. Soc. 2003, 125, 2830. g) J. H. Park, Y. T. Lim, O. O. Park, J. K. Kim, J.-W. Yu, Y. C. Kim, Chem. Mater. 2004, 16, 688. h) N. Cho, K. R. Choudhury, R. B. Thapa, Y. Sahoo, T. Ohulchanskyy, A. N. Cartwright, K. S. Lee, P. N. Prasad, Adv. Mater. 2007, 19, 232. i) A. A. R. Neves, A. Camposeo, R. Cingolani, D. Pisignano, Adv. Funct. Mater. 2008, 18, 751 .

[6] L. Pang, Y. Shen, K. Tetz, Y. Fainman, Opt. Express 2005, 13, 44.

[7] a) C. Ingrosso, V. Fakhfouri, M. Striccoli, A. Agostiano, A. Voigt, G. Gruetzner, M. L. Curri, J. Brugger, Adv. Funct. Mater. 2007, 17,
2009. b) C. Paquet, E. Kumacheva, Adv. Funct. Mater. 2007, 17, 3105 .

[8] a) V. C. Sundar, H. J. Eisler, T. Deng, Y. T. Chan, E. L. Thomas, M. G. Bawendi, Adv. Mater. 2004, 16, 2137. b) M. Tamborra, M. Striccoli, M. L. Curri, J. A. Alducin, D. Mecerreyes, J. A. Pomposo, N. Kehagias, V. Reboud, C. M. Sotomayor Torres, A. Agostiano, Small 2007, 3, 822. c) V. Reboud, N. Kehagias, C. M. Sotomayor Torres, M. Zelsmann, M. Striccoli, M. L. Curri, A. Agostiano, M. Tamborra, M. Fink, F. Reuther, G. Gruetzner, Appl. Phys. Lett. 2007, 90, 011115.

[9] a) K. Y. Suh, Y. S. Kim, H. H. Lee, Adv. Mater. 2001, 13, 1386. b) D. Pisignano, L. Persano, G. Gigli, R. Cingolani, F. Babudri, G. M. Farinola, F. Naso, Appl. Phys. Lett. 2004, 84, 1365.

[10] a) Y. Xia, G. M. Whitesides, Angew. Chem. 1998, 37, 550. b) H. Schmid, B. Michel, Macromolecules 2000, 33, 3042.

[11] S. Y. Chou, P. R. Krauss, P. J. Renstrom, Science 1996, 272, 85.

[12] A. Bietsch, B. Michel, J. Appl. Phys. 2000, $88,4310$.

[13] T. M. Squires, S. R. Quake, Rev. Mod. Phys. 2005, 77, 977.

[14] a) H. Vogel, Phys. Z 1921, 22, 645. b) G. S. Fulcher, J. Am. Ceram. Soc. 1925, 8, 339. c) G. Tamman, W. Hesse, Z. Anorg. Allg. Chem. 1926, 156, 245. d) K. L. Ngai, D. J. Plazek, Physical Properties of Polymers Handbook (Ed: J. E. Mark ), American Institute of Physics Press, Woodbury, New York 1996, pp. 72-152.

[15] M. Yan, L. J. Rothberg, F. Papadimitrakopoulos, M. E. Galvin, T. M. Miller, Phys. Rev. Lett. 1994, 73, 744.

[16] As low-viscosity prepolymers (i.e. with $\eta \cong 0.15 \mathrm{~Pa}$ s) typically exhibit penetration speed, $\mathrm{d} z / \mathrm{d} t$, of the order of $10^{2}-10^{3} \mu \mathrm{m} \mathrm{s}^{-1}$ at the entrance of $10 \mu \mathrm{m}$-wide microchannels see D. Pisignano, F. Di Benedetto, L. Persano, G. Gigli, R. Cingolani, Langmuir 2004, 20, 4802, corresponding to shear-rates of roughly $(\mathrm{d} z / \mathrm{d} t) / R=10-10^{2} \mathrm{~s}^{-1}$, we expect for our more viscous systems $\left(\eta>10^{3} \mathrm{Pas}\right)$, shear rates well below $10 \mathrm{~s}^{-1}$

[17] a) R. Valsecchi, M. Viganò, M. Levi, S. Turri, J. Appl. Polym. Sci. 2006, 102, 4484. b) B. Hoffmann, J. Kressler, G. Stöppelmann, C. Friedrich, G.-M. Kim, Colloid Polym. Sci. 2000, 278, 629. c) H. J. Choi, S. G. Kim, Y. H. Hyun, M. S. Jhon, Macromol. Rapid Commun. 2001, 22, 320. d) N. Artzi, Y. Nir, M. Narkis, A. Siegmann, J. Polym. Sci. B: Polym. Phys. 2002, 40, 1741. e) O. Meincke, B. Hoffmann, C. Dietrich, C. Friedrich, Macromol. Rapid Commun. 2003, 204, 823. f) H. B. Kim, J. S. Choi, C. H. Lee, S. T. Lim, M. S. Jhon, H. J. Choi, Eur. Polym. J. 2005, 41, 679. g) A. P. Shapiro, R. F. Probstein, Phys. Rev. Lett. 1992, 68,1422 .

[18] a) T.-Q. Nguyen, B. J. Schwartz, R. D. Schaller, J. C. Johnson, L. F. Lee, L. H. Haber, R. J. Saykally, J. Phys. Chem. B 2001, 105, 5153. b) J. M. Lupton, M. R. Craig, E. W. Meijer, Appl. Phys. Lett. 2002, 80, 4489. c) E. J. W. List, R. Guentner, P. Scanducci de Freitas, U. Scherf, $A d v$. Mater. 2002, 14, 374

[19] N. C. Greenham, I. D. W. Samuel, G. R. Hayes, R. T. Phillips, Y. A. R. R. Kessener, S. C. Moratti, A. B. Holmes, R. H. Friend, Chem. Phys. Lett. 1995, 241, 89

[20] a) B. J. Matterson, J. M. Lupton, A. F. Safonov, M. G. Salt, W. L. Barnes, I. D. W. Samuel, Adv. Mater. 2001, 13, 123. b) G. A. Turnbull, P. Andrews, M. J. Jory, W. L. Barnes, I. D. W. Samuel, Phys. Rev. B 2001, 64, 125122. c) E. Mele, F. Di Benedetto, L. Persano, R. Cingolani, D. Pisignano, Nano Lett. 2005, 5, 1915.

[21] G. Strobl, The Physics of Polymers, Springer, Berlin, Germany 1997, pp. 192-244. 